\title{
On the Nature of $X(4260)$
}

June 1, 2021

\author{
L. Y. DAi, ${ }^{\dagger}$ M Meng Shi, ${ }^{\dagger}$ GuAng-Yi TAng, ${ }^{\dagger}$ H. Q. ZhenG ${ }^{\dagger}, \star 2$ \\ $\dagger$ Department of Physics and State Key Laboratory of Nuclear Physics and Technology, Peking \\ University, Beijing 100871, P. R. China \\ * Collaborative Innovation Center of Quantum Matter, Beijing, Peoples Republic of China
}

\begin{abstract}
We study the property of $X(4260)$ resonance by re-analyzing all experimental data available, especially the $e^{+} e^{-} \rightarrow J / \psi \pi^{+} \pi^{-}, \omega \chi_{c 0}$ cross section data. The final state interactions of the $\pi \pi, K \bar{K}$ couple channel system are also taken into account. A sizable coupling between the $X(4260)$ and $\omega \chi_{c 0}$ is found. The inclusion of the $\omega \chi_{c 0}$ data indicates a small value of $\Gamma_{e^{+} e^{-}}=23.30 \pm 3.55 \mathrm{eV}$.
\end{abstract}

\section{Introduction}

The $X(4260)$ (previously called $Y(4260)$ ) resonance has been found by BaBar Collaboration in initial-state radiation (ISR) process, $e^{+} e^{-} \rightarrow \gamma_{I S R} J / \psi \pi^{+} \pi^{-}$, in year 2005 [1], and has been confirmed by CLEO [2] and Belle [3] Collaborations, respectively. In Ref. [4, the mass and width of this resonance are given with $M=4251 \pm 9 \mathrm{MeV}$ and $\Gamma=120 \pm 12 \mathrm{MeV}$, respectively. Furthermore, $\Gamma_{e e} \times \operatorname{Br}(J / \psi \pi \pi)=9.7 \pm 1.1 \mathrm{eV}$ [3] or $9.2 \pm 1.5 \mathrm{eV}$ [5]. In theory aspect, the structure of $X(4260)$ is very interesting, since it is generally thought that there are not enough unassigned vector states in charmonium spectrum (including the recently reported $Y(4360)$, $X(4630) / Y(4660)$ states $)$, also the masses are inconsistent with naive quark model predictions $\underline{6}$ - the only such $1^{--}$states expected up to $4.4 \mathrm{GeV}$ are $1 \mathrm{~S}, 2 \mathrm{~S}, 1 \mathrm{D}, 3 \mathrm{~S}, 2 \mathrm{D}$ and $4 \mathrm{~S}$, and they seem to be well established [7. The situation is depicted in Figure 1] It is noticed that above $D \bar{D}$ threshold the number of $1^{--}$states given by quark model prediction is inconsistent with that given by experiments. One tends to believe that the discrepancy between the naive quark model prediction and the observed spectrum is ascribed, at least partially, to the existence of many open charm thresholds, since the latter will distort the spectrum. The situation is depicted in Figure2. Because of the situation as described above many theoretical papers have been devoted to the investigation on $X(4260)$. In the literature, many models have been made, e.g., $\chi_{c 0} \rho^{0}$ molecule [8], $\omega \chi_{c 1}$ molecule [9], $c \bar{c} g$ hybrid state [10]14, $\Lambda_{c} \bar{\Lambda}_{c}$ bayronium [15, $D_{1} \bar{D}$ or $D_{0} \bar{D}_{0}^{*}$ molecule [1618], non-resonant explanation [19,20], etc.. Besides, the tetraquark state explanation is also very intersting [21 26], especially when two resonances, $Z_{c}(3900)$ and $Z_{c}(4025)$, are recently found in $J / \psi \pi$ and $D^{(*)} \bar{D}^{(*)}$ channels in $e^{+} e^{-}$annihilation near $4.26 \mathrm{GeV}$ by BESIII Collaboration [27, and confirmed by Belle 28] and CLEO 29] Collaborations. However the open charm channels such as $\bar{D} D^{*}, D \bar{D}^{*}, D^{*} \bar{D}^{*}$ are not found in the final states of $\mathrm{X}(4260)$ decays 30 , making the property of $X(4260)$ to be more mysterious.

The present authors have also studied the $X(4260)$ issue in the previous edition of the present paper (Preprint arXiv:1206.6911v2, herewith denoted as V1). Through a careful analysis to experimental data available, it is found that the $X(4260)$ has a sizable coupling to $\omega \chi_{c 0}$ channel, but not to other (nearby) channels. Inspired by our result, a recent experimental analysis 33,34 shows that there is indeed a sizable $\omega \chi_{c 0}$ final state signal in $e^{+} e^{-}$collision at around $4.26 \mathrm{GeV}$,

\footnotetext{
${ }^{1}$ present address: Center for Exploration of Energy and Matter, 2401 Milo B. Sampson Lane, Bloomington, IN 47408 , USA.

${ }^{2}$ e-mail address: zhenghq@pku.edu.cn
} 


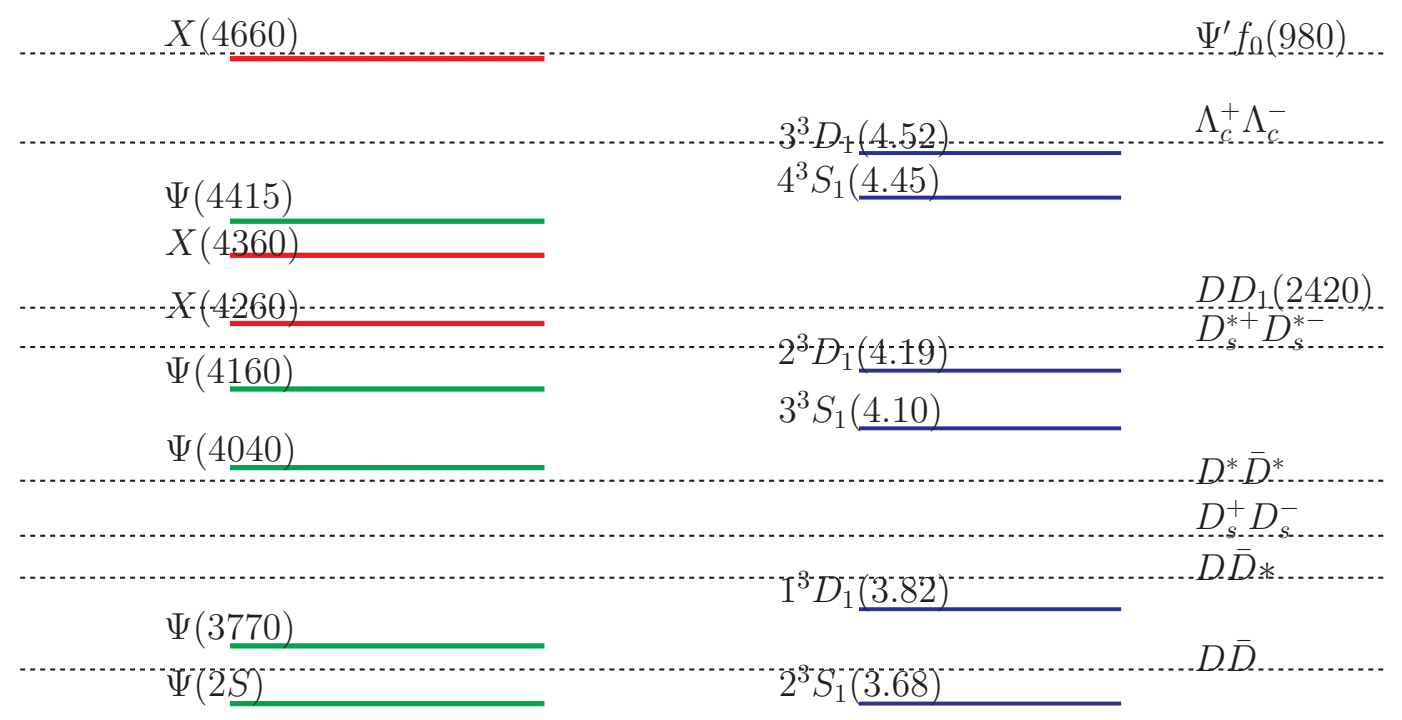

$\Psi(1 S)$

$1^{--}$particle
$1^{3} S_{1}(3.1)$

quark model threshold

Figure 1: $\mathrm{X}(4260)$ and nearby resonances from naive quark model calculation $[6]$.

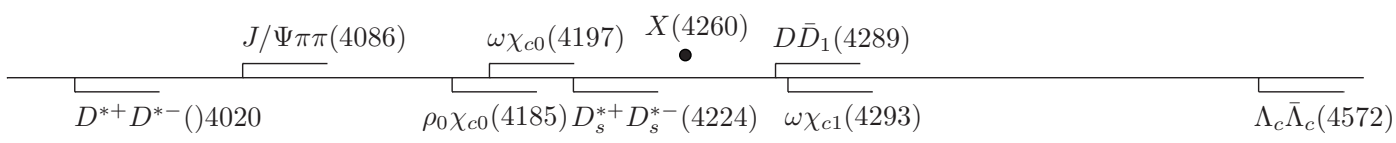

Figure 2: Location of X(4260) and nearby thresholds.

which hints that the $X(4260)$ may have a large coupling to $\omega \chi_{c 0}$ - though it is not totally clear whether the $\omega \chi_{c 0}$ is from the continuum spectrum or the $\mathrm{X}(4260)$ resonance, or from both. Furthermore, the cross section of $h_{c} \pi \pi$ channel [35] is also measured at this energy implying that the X(4260) may also couple to it. Because of all these new observations, we have an urge to upgrade the work of V1. In the present paper, we continue the preceding analysis by including the $\omega \chi_{c 0}$ (and also $h_{c} \pi \pi$ ) cross section data, and we find that the major qualitative conclusion of V1 still holds, that is $X(4260)$ couples significantly to $\omega \chi_{c 0}$ but not to other nearby thresholds. Furthermore, we find that the $X(4260)$ resonance is likely to maintain a small $e^{+} e^{-}$ width, thanks to the new $\omega \chi_{c 0}$ data.

The paper is organized as the following: In section 2 we review on theoretical tools we use in this paper, where special emphasis is made on the final state interactions between pions and kaons. In section 3 we give a detailed description to our numerical fit program with two scenarios, one does not include the $\omega \chi_{c 0}$ cross section data [33, 34, and the other one includes. Both of them take into account the effect of the possible $h_{c} \pi \pi$ decay channel. The pole locations of the $X(4260)$ propagator are also searched for. Finally conclusions and physical discussions on the present analysis is given in section 4. 


\section{Theoretical Discussions on $e^{+} e^{-} \rightarrow J / \psi \pi \pi$}

\subsection{Effective Lagrangian Describing $e^{+} e^{-} \rightarrow J / \psi \pi \pi$ Interactions}

Assuming that the $X(4260)$ is a $J^{P C}=1^{--}$chiral singlet particle. The transition operator between photon and $X(4260)$ is as the following:

$$
\mathcal{L}_{\gamma X}=g_{0} X_{\mu \nu} F^{\mu \nu}
$$

where we use the anti-symmetric representation $X_{\mu \nu}$ to describe the $1^{--}$state $X(4260)$, and $F_{\mu \nu}$ denotes the photon field strength. Notice that in the present notation, one has

$$
\Gamma_{e^{+} e^{-}}=\frac{4 \alpha}{3} \frac{g_{0}^{2}}{M_{X}}
$$

where we have neglected the electron-positron masses. For $X(4260)$ decay, the following effective lagrangian is used, which is accurate in the leading order in the expansion in terms of $\pi$ momentum in the center of mass frame of $\pi \pi$ system:

$$
\mathcal{L}_{X \psi P P}=h_{1} X_{\mu \nu} \psi^{\mu \nu}<u_{\alpha} u^{\alpha}>+h_{2} X_{\mu \nu} \psi^{\mu \nu}<\chi_{+}>+h_{3} X_{\mu \alpha} \psi^{\mu \beta}<u_{\beta} u^{\alpha}>
$$

where anti-symmetric representation $\psi^{\mu \nu}$ describes $J / \psi$. Up to $O\left(p_{\pi}^{2}\right)$ level, in Eq. (3) there exist only three independent interaction terms with coefficients $h_{1}, h_{2}$ and $h_{3}$. Further, $u_{\mu}=$ $i\left(u^{+} \partial_{\mu} u-u \partial_{\mu} u^{+}\right)$and

$$
u=\exp \left\{i \frac{\Phi}{\sqrt{2} F_{\pi}}\right\}
$$

is the parametrization of the pseudo-goldstone octet:

$$
\Phi=\left(\begin{array}{ccc}
\frac{1}{\sqrt{2}} \pi^{0}+\frac{1}{\sqrt{6}} \eta_{8} & \pi^{+} & K^{+} \\
\pi^{-} & -\frac{1}{\sqrt{2}} \pi^{0}+\frac{1}{\sqrt{6}} \eta_{8} & K^{0} \\
K^{-} & \bar{K}^{0} & -\frac{2}{\sqrt{6}} \eta_{8}
\end{array}\right) .
$$

The chiral symmetry breaking term with coefficient $h_{2}$ in Eq. (3) reads,

$$
\chi_{+}=u^{+} \chi u^{+}+u \chi^{+} u, \quad \chi=2 B_{0} \operatorname{diag}\left(m_{u}, m_{d}, m_{s}\right) .
$$

Parameters $F_{\pi}$ and $B_{0}$ can be fixed phenomenologically: $F_{\pi} \approx 92.4 \mathrm{MeV}$ and $<0|\psi \bar{\psi}| 0>=$ $-F^{2} B_{0}\left[1+O\left(m_{q}\right)\right]$. The Eq. (3) can also be rewritten in an explicit form,

$$
\begin{aligned}
\mathcal{L}_{1}= & \frac{4 h_{1}}{F_{\pi}^{2}} X_{\mu \nu} F^{\mu \nu}\left(\partial_{\rho} \pi^{+} \partial^{\rho} \pi^{-}+\frac{1}{2} \partial_{\rho} \pi^{0} \partial^{\rho} \pi^{0}+\partial_{\rho} K^{+} \partial^{\rho} K^{-}+\partial_{\rho} K^{0} \partial^{\rho} \bar{K}^{0}+\frac{1}{2} \partial_{\rho} \eta \partial^{\rho} \eta\right) \\
\mathcal{L}_{2}= & -\frac{4 h_{2}}{F_{\pi}^{2}} X_{\mu \nu} F^{\mu \nu}\left(m_{\pi}^{2} \pi^{+} \pi^{-}+\frac{1}{2} m_{\pi}^{2} \pi^{0} \pi^{0}+m_{K}^{2} K^{+} K^{-}+m_{K}^{2} K^{0} \bar{K}^{0}+\left(\frac{2}{3} m_{K}^{2}-\frac{1}{6} m_{\eta}^{2}\right) \eta \eta\right) \\
\mathcal{L}_{3}= & \frac{4 h_{3}}{F_{\pi}^{2}} X_{\mu \alpha} F^{\mu \beta}\left(\frac{1}{2} \partial_{\beta} \pi^{+} \partial^{\alpha} \pi^{-}+\frac{1}{2} \partial_{\beta} \pi^{-} \partial^{\alpha} \pi^{+}+\frac{1}{2} \partial_{\beta} \pi^{0} \partial^{\alpha} \pi^{0}+\frac{1}{2} \partial_{\beta} K^{+} \partial^{\alpha} K^{-}+\frac{1}{2} \partial_{\beta} K^{-} \partial^{\alpha} K^{+}\right. \\
& \left.+\frac{1}{2} \partial_{\beta} K^{0} \partial^{\alpha} \bar{K}^{0}+\frac{1}{2} \partial_{\beta} \bar{K}^{0} \partial^{\alpha} K^{0}+\frac{1}{2} \partial_{\beta} \eta^{0} \partial^{\alpha} \eta^{0}\right)
\end{aligned}
$$

\subsection{Kinematics and Tree Level Amplitudes}

We denote the momenta of $e^{-}, e^{+}, X(4260), J / \psi, \pi^{+}$and $\pi^{-}$as $q_{1}, q_{2}, q, q_{0}, q^{+}$and $q^{-}$ respectively, see in Fig. 3. The polarization of $J / \psi$ is represented as $\epsilon_{\psi}$, and $k_{ \pm}=q^{+} \pm q^{-}$. Then one has the following relations:

$$
\begin{aligned}
& s \equiv k_{+}^{2}, \\
& k_{-}^{2}=-s \rho(s)^{2}=4 m_{\pi}^{2}-s, \\
& q_{0}^{2}=M_{J / \psi}^{2}, k_{+} \cdot k_{-}=0, \\
& k_{+} \cdot q_{0}=\frac{1}{2}\left(q^{2}-M_{J / \psi}^{2}-s\right),
\end{aligned}
$$




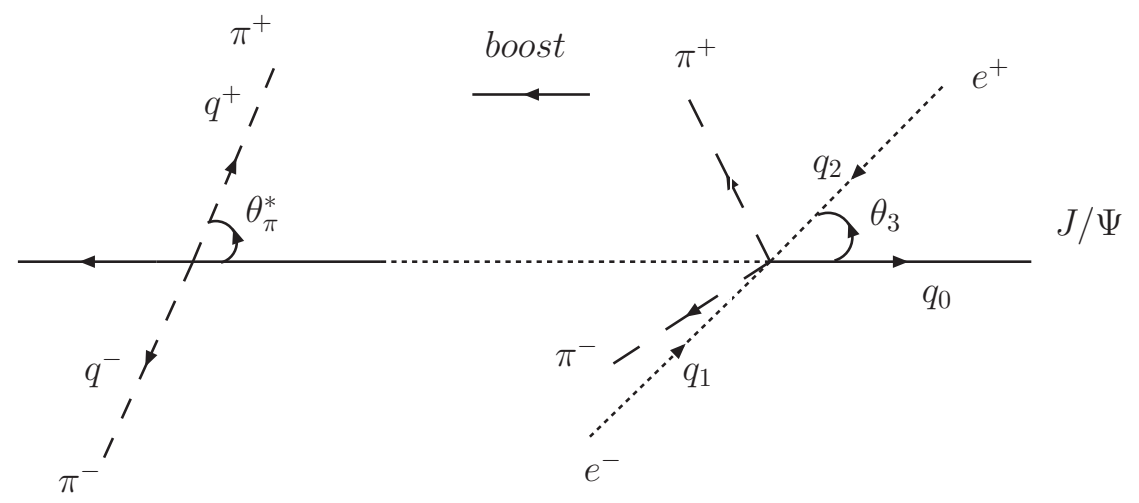

Figure 3: A depiction of kinematics.

where $\rho(s)=\sqrt{1-\frac{4 m_{\pi}^{2}}{s}}$. The amplitude of $X \rightarrow J / \psi \pi^{+} \pi^{-}$process at tree level is

$$
\begin{aligned}
i \mathcal{A}^{\text {tree }}= & \frac{i 4 e g_{0}}{M_{J / \psi} F_{\pi}^{2} q^{2} D_{X}\left(q^{2}\right)} \bar{v}\left(q_{1}, s\right) \gamma_{\lambda} u\left(q_{2}, s^{\prime}\right)\left\{\left[4 h_{1} \frac{1}{2}\left(s-2 m_{\pi}^{2}\right)+4 h_{2} m_{\pi}^{2}\right]\left(q^{0} \cdot q \epsilon_{\psi}^{\lambda}-q \cdot \epsilon_{\psi} q^{0 \lambda}\right)\right. \\
& +\frac{1}{2} h_{3}\left[-q_{\alpha}^{0} q \cdot \epsilon_{\psi}\left(k_{+}^{\lambda} k_{+}^{\alpha}-k_{-}^{\lambda} k_{-}^{\alpha}\right)+\epsilon_{\psi}^{\lambda} q_{\alpha}^{0} q_{\beta}\left(k_{+}^{\alpha} k_{+}^{\beta}-k_{-}^{\alpha} k_{-}^{\beta}\right)\right. \\
& \left.\left.-q^{0 \lambda} q_{\alpha} \epsilon_{\psi \beta}\left(k_{+}^{\alpha} k_{+}^{\beta}-k_{-}^{\alpha} k_{-}^{\beta}\right)+q^{0} \cdot q \epsilon_{\psi \alpha}\left(k_{+}^{\lambda} k_{+}^{\alpha}-k_{-}^{\lambda} k_{-}^{\alpha}\right)\right]\right\}
\end{aligned}
$$

where $\alpha, \beta, \lambda$ are Lorentz indices and $D_{X}\left(q^{2}\right)$ is the denominator of the $X(4260)$ propagator which will be discussed latter.

Following the helicity amplitude decomposition method [36] and choosing the basis of tensors

$$
\begin{aligned}
\tilde{t}^{(0)} & =1, \\
\tilde{t}^{(1)} & =k_{-}^{\mu}, \\
\tilde{t}^{(2)}=k_{-}^{\mu} k_{-}^{\nu}-\frac{1}{3} k_{-}^{2} \tilde{g}^{\mu \nu} & ,\left(\tilde{g}^{\mu \nu}=g^{\mu \nu}-\frac{k_{+}^{\mu} k_{+}^{\nu}}{k_{+}^{2}}\right),
\end{aligned}
$$

it is easy to separate the $S$-wave and $D$-wave components of $\pi \pi$ system. The overall $S$-wave amplitude reads,

$$
\begin{aligned}
i \mathcal{A}_{s}^{\text {tree }}= & \frac{i 4 e g_{0}}{M_{J / \psi} F_{\pi}^{2} q^{2} D_{X}\left(q^{2}\right)} \bar{v}\left(q_{1}, s\right) \gamma_{\lambda} u\left(q_{2}, s^{\prime}\right) \epsilon_{\psi \omega}\left\{\left[4 h_{1} \frac{1}{2}\left(s-2 m_{\pi}^{2}\right)+4 h_{2} m_{\pi}^{2}\right]\left(q^{0} \cdot q g^{\lambda \omega}-q^{\omega} q^{O \lambda}\right)\right. \\
& +\frac{1}{2} h_{3}\left[-\frac{1}{3} \rho^{2}(s) q_{\lambda}^{0} q^{\omega} s+\left(1-\frac{1}{3} \rho^{2}(s)\right) k_{+}^{\lambda} q^{\omega} q^{0} \cdot k_{+}+\frac{1}{3} \rho^{2}(s) q^{0} \cdot q g^{\lambda \omega} s\right. \\
& -\left(1-\frac{1}{3} \rho^{2}(s)\right) g^{\lambda \omega} k_{+} \cdot q k_{+} \cdot q^{0}-\frac{1}{3} \rho^{2}(s) q_{\lambda}^{0} q^{\omega} s+\left(1-\frac{1}{3} \rho^{2}(s)\right) k_{+}^{\lambda} q^{O \omega} q \cdot k_{+} \\
& \left.\left.+\frac{1}{3} \rho^{2}(s) q^{0} \cdot q g^{\lambda \omega} s-\left(1-\frac{1}{3} \rho^{2}(s)\right) k_{+}^{\lambda} k_{+}^{\omega} q \cdot q^{0}\right]\right\},
\end{aligned}
$$

whereas the $D$-wave part is then

$$
\begin{aligned}
i \mathcal{A}_{d}^{\text {tree }} & =\frac{i 4 e g_{0}}{M_{J / \psi} F_{\pi}^{2} q^{2} D_{X}\left(q^{2}\right)} \bar{v}\left(q_{1}, s\right) \gamma_{\lambda} u\left(q_{2}, s^{\prime}\right) \epsilon_{\psi}^{\omega} \\
& \frac{1}{2} h_{3}\left(t_{2}^{\lambda \alpha} q_{\alpha}^{0} q_{\omega}-t_{2}^{\alpha \beta} g_{\lambda \omega} q_{\alpha}^{0} q_{\beta}+t_{2}^{\alpha \beta} g_{\beta \omega} q_{\lambda}^{0} q_{\alpha}-t_{2}^{\lambda \alpha} g_{\alpha \omega} q^{0} \cdot q\right) \\
& \equiv \frac{2 e g_{0} h_{3}}{F_{\pi}^{2} M_{J / \psi} q^{2} D_{X}\left(q^{2}\right)} B_{d} .
\end{aligned}
$$


The standard Breit-Wigner type of $\mathrm{X}(4260)$ propagator is parametrized as $D_{X}\left(q^{2}\right)=M_{X}^{2}-$ $q^{2}-i M_{X} \Gamma_{X}\left(q^{2}\right)$, where $\Gamma_{X}\left(q^{2}\right)$ is the total decay width including partial width of all possible channels that will be discussed latter.

Since the $D$-wave contribution is proportional to the 4 th power of the kinematic factor $\rho(s)$, it is highly suppressed comparing with the $S$-wave contribution. Through numerical studies it is shown that the $D$-wave contribution is roughly less than $1 \%$ of the total decay rate, therefore we will not include it in the fitting process in this work.

\subsection{Final State Interactions(FSI)}

The tree level amplitude as described in section 2.2 is not sufficient to describe the $X \rightarrow$ $J / \psi \pi \pi$ decay process, since the $\pi \pi$ system undergoes strong final state interactions (FSI), especially in $I J=00$ channel. To include FSI, the following decay amplitude is proposed [37]:

$$
\begin{aligned}
& \mathcal{A}_{1}=\mathcal{A}_{1}^{\text {tree }} \alpha_{1}(s) T_{11}(s)+\mathcal{A}_{2}^{\text {tree }} \alpha_{2}(s) T_{21}(s), \\
& \mathcal{A}_{2}=\mathcal{A}_{1}^{\text {tree }} \alpha_{1}(s) T_{12}(s)+\mathcal{A}_{2}^{\text {tree }} \alpha_{2}(s) T_{22}(s),
\end{aligned}
$$

where subscripts 1,2 denote $\pi \pi$ and $K \bar{K}$ final states, respectively. For $A_{2}^{\text {tree }}$ (the $K \bar{K}$ amplitude), one only needs to change the $m_{\pi}$ into $m_{K}$ in $A_{1}^{\text {tree }}$ (the $\pi \pi$ amplitude). Especially $T_{11}, T_{12}$ and $T_{22}$ represent $\pi \pi \rightarrow \pi \pi, \pi \pi \rightarrow K \bar{K}, K \bar{K} \rightarrow K \bar{K}$ scattering amplitudes, respectively. Functions $\alpha_{i}$ are mild polynomials which place the role to offset the 'left hand' cuts on the complex $s$ plane in amplitude $T$ that would not appear in function $\mathcal{A}$. Expressions in Eq. (13) are remarkable in the sense that the unitarity relations are automatically satisfied. The decay amplitudes $\mathcal{A}_{i}$, as an analytic function of $s$, obey:

$$
\begin{aligned}
\operatorname{Im} \mathcal{A}_{1} & =\mathcal{A}_{1}^{*} \rho_{1} T_{11}+\mathcal{A}_{2}^{*} \rho_{2} T_{21}, \\
\operatorname{Im} \mathcal{A}_{2} & =\mathcal{A}_{1}^{*} \rho_{1} T_{12}+\mathcal{A}_{2}^{*} \rho_{2} T_{22} .
\end{aligned}
$$

In $\alpha_{1}(s)$ an additional pole term is added:

$$
\alpha_{1}(s)=\frac{c_{0}^{(1)}}{s-s_{A}}+c_{1}^{(1)}+c_{2}^{(1)} s+\cdots,
$$

where $s_{A}$ represents the Adler zero of $T_{11}$. The role of the pole term is to cancel the Adler zero hidden in $T_{11}$ but not welcome in $A$ [37. An advantage of the pole term in Eq. (15) is that, by appropriately choosing coefficient $c_{0}^{(1)}$ as $\lim _{s \rightarrow s_{A}} \frac{c_{0}^{(1)}}{s-s_{A}} T_{11}(s)=1$, it guarantees

$$
\mathcal{A}_{1}=\mathcal{A}_{1}^{\text {tree }}+O\left(s^{2}\right)
$$

Up to the leading order in $\chi \mathrm{PT}$, one finds $c_{0}^{(1)}=16 \pi F_{\pi}^{2}$ and $s_{A}=m_{\pi}^{2} / 2$ in Isospin $0 \mathrm{~S}$ wave. However in [1,1] Matrix Padé amplitude, $s_{A}=(0.490-0.008 i) m_{\pi}^{2}$ and we fix $c_{0}^{(1)}=$ $(0.330-0.001 i) \mathrm{GeV}^{2}=(0.779-0.002 i) 16 \pi F_{\pi}^{2} \cdot 3$

\begin{tabular}{|c|c|c|c|c|c|c|}
\hline$L_{1}$ & $L_{2}$ & $L_{3}$ & $L_{4}$ & $L_{5}$ & $2 L_{6}+L_{8}$ & $2 L_{7}+L_{8}$ \\
\hline 0.881 & 1.029 & -3.803 & 0.176 & 1.111 & 1.123 & 0.392 \\
\hline
\end{tabular}

Table 1: Low energy constants from couple channel Padé amplitudes. Here these parameters are refitted and are slightly different from Ref. 38. The unit is $10^{-3}$.

In V1, three different representations of $T$ matrices were used for the fit (coupled channel Padé approximation [38, K-matrix unitarization [39] and the PKU representation [40] ), however not much difference was obtained. Therefore we only keep the Padé amplitude in this work to perform the fit to the $\pi \pi$ invariant mass spectrum. In Table 1 we list the low energy constant: 4). For more details about these $\mathrm{T}$ matrices, we refer to the original literature.

\footnotetext{
${ }^{3}$ The Adler zero moves to the complex plane because the existence of the left hand cut $\left(-\infty, 4 m_{K}^{2}-4 m_{\pi}^{2}\right]$ of $T_{22}$, which has been taken into $T_{11}$ due to Matrix Padé approximation. And so on for that of $c_{0}^{1}$.

${ }^{4} \mathrm{We}$ noticed that for these LECs there is a difference between ours 38 $\left(L_{i}^{O}\right)$ and that in the earlier work [4] $\left(L_{i}^{P}\right): \quad L_{i}^{P}=L_{i}^{O}+\frac{\Gamma_{i}}{32 \pi^{2}}$. It is because we calculated in $\overline{M S}$ and theirs in $\overline{M S}-1$. To compare with their work we need to transform our LECs into $L_{i}^{P}$, which has been incorrectly transferred in 38 and we correct it here.
} 


\section{Numerical Analysis}

\subsection{The Experimental Data and Fit Process}

Once the $X(4260) \rightarrow J / \psi \pi \pi$ amplitude is calculated from Eq. (13) in above section, one obtains the decay width $\Gamma_{J / \psi \pi \pi}$, the cross section $e^{+} e^{-} \rightarrow X(4260) \rightarrow J / \psi \pi \pi$ and the $\pi \pi$ invariant mass spectrum. In this subsection we focus on how to write down the correct form of denominator of the $X(4260)$ propagator.

Beside the $J / \psi \pi \pi$ channel indicated by the experiment, the $\mathrm{X}(4260)$ may also decay into $h_{c} \pi \pi$, and there are other nearby thresholds close to $\mathrm{X}(4260)$, such as $\chi_{c 0} \omega(4197 \mathrm{MeV}), D_{s}^{*+} \bar{D}_{s}^{*-}$ $(4224 \mathrm{MeV}), D^{-} D_{1}^{+}(2420)(4291 \mathrm{MeV}), \chi_{c 1} \omega(4293 \mathrm{MeV})$, etc.. It is possible that $\mathrm{X}(4260)$ couples to all these channels. Therefore a careful way is to write down the denominator of the $X(4260)$ propagator as:

$$
D_{X}\left(q^{2}\right)=M_{X}^{2}-q^{2}-i \sqrt{q^{2}} \Gamma\left(q^{2}\right),
$$

where $\Gamma_{X}\left(q^{2}\right)$ consists of all partial widths,

$$
\Gamma_{X}\left(q^{2}\right)=\Gamma_{J / \psi \pi \pi}\left(q^{2}\right)+\Gamma_{h_{c} \pi \pi}+g_{\omega \chi_{c 0}} k_{\omega \chi_{c 0}}+g_{D_{s}^{*} D_{s}^{*}} k_{D_{s}^{*} D_{s}^{*}}^{3}+g_{D D_{1}} k_{D D_{1}}+g_{\omega \chi_{c 1}} k_{\omega \chi_{c 1}}+\Gamma_{0} .
$$

Here $\Gamma_{J / \psi \pi \pi}\left(q^{2}\right)$ is calculated from the above amplitude of $X(4260) \rightarrow J / \psi \pi \pi$, and $k_{\omega \chi_{c 0}}$, $k_{D_{s}^{*} D_{s}^{*}}, k_{D D_{1}}, k_{\omega \chi_{c 1}}$ are the 3 -momentum of $\omega \chi_{c 0}, D_{s}^{*} D_{s}^{*}, D D_{1}, \omega \chi_{c 1}$ in the $X(4260)$ rest frame, respectively. The $D_{s}^{*} D_{s}^{*}$ channel begins with a P-wave coupling therefore it depends on $3 \mathrm{rd}$ order momentum, and the other three channels are of S-wave couplings and hence only depend on their 1st-order momentum. The possible rest partial widths are parameterized as a constant $\Gamma_{0}$. For $h_{c} \pi \pi$, since the channel momentum $k_{h_{c} \pi \pi}$ behaves like a constant near $q^{2}=M_{X}^{2}$, we parameterize $\Gamma_{h_{c} \pi \pi}$ as a constant, too. Notice that $\Gamma_{h_{c} \pi \pi} / \Gamma_{J / \psi \pi \pi}\left(M_{X}^{2}\right)$ is constrained by experiment. We discuss this point in more detail in the next section.

One may notice that $Z_{c} \pi$ is also a possible decay channel of the $\mathrm{X}(4260)$ but it is not considered in this work, since the contribution of $X(4260) \rightarrow Z_{c} \pi \rightarrow J / \psi \pi \pi$ can be absorbed into the $X J / \psi \pi \pi$ contact interaction in the Lagrangian Eq. (3). To confirm this viewpoint, we tested the contribution of $Z_{c} \pi$ with Breit-Wigner parametrization of $Z_{c}$, and found that there was not much difference in $J / \psi \pi \pi$ and $\pi \pi$ spectrums from that without its contribution. Therefore we exclude $Z_{c} \pi$ contribution in this paper and leave it for future work.

In the present work we fit the $\mathrm{X}(4260)$ line shape in the region of $4.15 \mathrm{GeV}<\sqrt{q^{2}}<4.47 \mathrm{GeV}$, where the data is from Ref. [3] (16 data points) and Ref. [5] (16 data points), see in Figure 5 a . The total cross section of $e^{+} e^{-} \rightarrow J / \psi \pi \pi$ is given by

$$
\sigma_{e^{+} e^{-} \rightarrow J / \psi \pi^{+} \pi^{-}}=\int_{s_{-}}^{s_{+}} \int_{t_{-}}^{t_{+}} \frac{{\overline{\left|\mathcal{A}_{1}\right|}}^{2} d s d t}{(2 \pi)^{3} 32\left(q^{2}\right)^{2}},
$$

where $t=\left(q-q^{-}\right)^{2}, \mathcal{A}_{1}$ is defined in Eq. 13 and the lower and upper limits are given as

$$
\begin{aligned}
& s_{-}=4 m_{\pi}^{2} \\
& s_{+}=\left(\sqrt{q^{2}}-M_{\Psi}\right)^{2} \\
& t_{ \pm}=\frac{1}{4 s}\left\{\left(q^{2}-M_{\Psi}^{2}\right)^{2}-\left[\lambda^{1 / 2}\left(q^{2}, s, M_{\Psi}^{2}\right) \mp \lambda^{1 / 2}\left(s, m_{\pi}^{2}, m_{\pi}^{2}\right)\right]^{2}\right\} \\
& \lambda(a, b, c)=(a-b-c)^{2}-4 b c .
\end{aligned}
$$

With Eq. (20), one finds out that for a larger $q^{2}$, the upper limit of $s$ becomes too large to insure the validity of the parametrization introduced in section 2.35. For $\pi \pi$ invariant mass spectrum we use the data given in figure $4 \mathrm{~b}$ of Ref. [3], corresponding to $\sqrt{q^{2}} \in[4.2,4.4] \mathrm{GeV}$ (17 data points), the data $\sqrt{q^{2}} \in[4.15,4.45] \mathrm{GeV}$ (41 data points) in Ref. [5], and the data $\sqrt{q^{2}}$ fixed at $4.26 \mathrm{GeV}$ (44 points) from the recent experiment 27. For the first set of data, a MC study of efficiency correction at $\sqrt{q^{2}}=4.26 \mathrm{GeV}$ is given in Ref. [3], and through a numerical

\footnotetext{
${ }^{5}$ For $\sqrt{q^{2}}=4.47 \mathrm{GeV}$, it corresponds to a value $\sqrt{s} \simeq 1.17 \mathrm{GeV}$ which is within the range that $T$ can provide a reasonable description.
} 
test we find that the efficiency curve is well reproduced by the $\pi \pi$ two body phase space up to a normalization constant, hence in our fit we simply use the two body phase space instead of the efficiency corrected one. We assume the other two sets of data maintain similar behavior. Summing up, there are totally 145 data points in $\pi^{+} \pi^{-}$invariant mass spectrum to be used.

Recently the cross section of $\omega \chi_{c 0}$ channel is measured in $[4.21,4.45] \mathrm{GeV}$ [33, 34, where a rough structure of the $X(4260)$ could be observed at $4.26 \mathrm{GeV}$. If we assume the events in Ref. [33, 34] all come from $X(4260)$ decay, then 9 data points can be used. Nevertheless it may be possible that these events come from continuum rather than from $X(4260)$, hence in our fit we carefully consider this possibility. That is, in the following section 3.2, the fit named Fit I, does not include the $\omega \chi_{c 0}$ cross section data, while in section 3.3 the $\omega \chi_{c 0}$ cross section data are fitted, named Fit II. It is found that Fit I does not differ much from the result of V1 (notice that here we include the $h_{c} \pi \pi$ channel), but Fit II, with the $\omega \chi_{c 0}$ data being included, violates the approximate 'scaling law' found in V1 and lead to a small value of $\Gamma_{e^{+}} e^{-}$.

Parameters needed in our fit are the following: Firstly, Eq. (11) and (3) describing the $\gamma-X$ transition and the tree level $X(4260) J / \psi \pi \pi(K \bar{K})$ interactions provide 4 parameters. Secondly, it is found that taking $\alpha_{1,2}(s)$ to be linear polynomials (except the Adler zero term) is already good enough for data fitting, hence the two $\alpha_{i}(s)$ ( $\mathrm{i}=1,2$ and each $\alpha_{i}(s)$ contains two parameters) contribute another 4 parameters. Thirdly the mass $M_{X}$ of the $X(4260)$ in the propagator and the coupling constant $g_{\omega \chi_{c 0}}$ bring another two parameters. Finally, there are three normalization factors $N_{1}, N_{2}$ and $N_{3}$ for the $\pi^{+} \pi^{-}$invariant mass spectrum of Belle, Babar and BESIII, respectively. After summing up there are totally 13 parameters.

We also studied the parameters $g_{D_{s}^{*} D_{s}^{*}}, g_{D D_{1}}, g_{\omega \chi_{c 1}}$ and $\Gamma_{0}$ through rather extensive numerical tests in different environments, and we found that the $g_{D_{s}^{*} D_{s}^{*}}, g_{D D_{1}}$ and $g_{\omega \chi_{c 1}}$ are always vanishingly small which suggests that the coupling of $\mathrm{X}(4260)$ to $D_{s}^{*} D_{s}^{*}, D D_{1}(2420)$ and $\omega \chi_{c 1}$ are negligible, compared to $J / \pi \pi, \omega \chi_{c 0}$ and $h_{c} \pi \pi$. Moreover, the parameter $\Gamma_{0}$ tends to vanish in all different fits and hence it can be ignored too 6 Therefore we will not include the couplings to $D_{s}^{*} D_{s}^{*}, D D_{1}(2420), \omega \chi_{c 1}$ and $\Gamma_{0}$ in our discussion from now on.

\subsection{Fit without $\omega \chi_{c 0}$ Data}

In this fit, named as Fit I, only the $J / \psi \pi \pi$ cross section and $\pi \pi$ invariant mass spectrum are included, but not the $\omega \chi_{c 0}$ cross section data which will be analyzed in the next section. About $h_{c} \pi \pi$ channel, however, on one hand, lacking of precision the rough shape is not appropriate to fit [42. On the other hand, it is not clear yet whether they are from the $X(4260)$ resonance or from the continuous background. Therefore we assume $h_{c} \pi \pi$ has an unknown width in the $X(4260)$ propagator, and it is assumed to be a constant because its threshold is far away from the $X(4260)$ resonance 7 and it is constrained by the width of $\Gamma_{J / \psi \pi \pi}\left(q^{2}\right)$ as the following relation

$$
\Gamma_{h_{c} \pi \pi}=R \times\left.\Gamma_{J / \psi \pi \pi}\left(q^{2}\right)\right|_{q=4.26 \mathrm{GeV}},
$$

where the coefficient $R=0.66$ is a rough estimation from the ratio of $\sigma_{h_{c} \pi \pi} / \sigma_{J / \psi \pi \pi}$ at $q=$ $4.26 \mathrm{GeV}$ 35. Of course, we will also test the fits with different $R$ value ranging from 0 to 0.66 .

When fit to the $J / \psi \pi \pi$ cross section and the $\pi \pi$ invariant mass, we found that the value of $g_{0}$ has large uncertainty. Since it characterizes the transition between $X(4260)$ and the photon field, or $\Gamma_{X \rightarrow e^{+} e^{-}}$according to Eq. (2), it should not be too large to avoid the upper bound established by an analysis on the BES experiment: Ref. [43] gives $\Gamma_{X \rightarrow e^{+} e^{-}}<420 \mathrm{eV}$, or most

\footnotetext{
${ }^{6}$ It is exactly for this reason we will band the $h_{c} \pi \pi$ width with the $J / \Psi \pi \pi$ width with a ratio $R$ (see Eq. (23)), otherwise the fit program will tend to destroy it.

${ }^{7}$ In fact, the momentum-dependent width of $h_{c} \pi \pi$ channel with form factor is also tested in the fitting as following,

$$
\Gamma_{h_{c} \pi \pi}\left(q^{2}\right) \sim \int \frac{\sqrt{\left(q^{2}-\left(\sqrt{s}+m_{h_{c}}\right)^{2}\right)\left(q^{2}-\left(\sqrt{s}-m_{h_{c}}\right)^{2}\right)} \sqrt{s-4 m_{\pi}^{2}}}{4 q s} d s d \Omega
$$

and

$$
\Gamma_{X}\left(q^{2}\right)=\left(\Gamma_{J / \psi \pi \pi}\left(q^{2}\right)+\Gamma_{h_{c} \pi \pi}\left(q^{2}\right)\right) \exp \left(\frac{q^{2}-M_{X}^{2}}{\Lambda^{2}}\right)+g_{\omega \chi_{c 0}} k_{\omega \chi_{c 0}}+\cdots
$$

This fitting result is always similar with the constant width of $h_{c} \pi \pi$, therefore only the constant $\Gamma_{h_{c} \pi \pi}$ is shown in the text.
} 


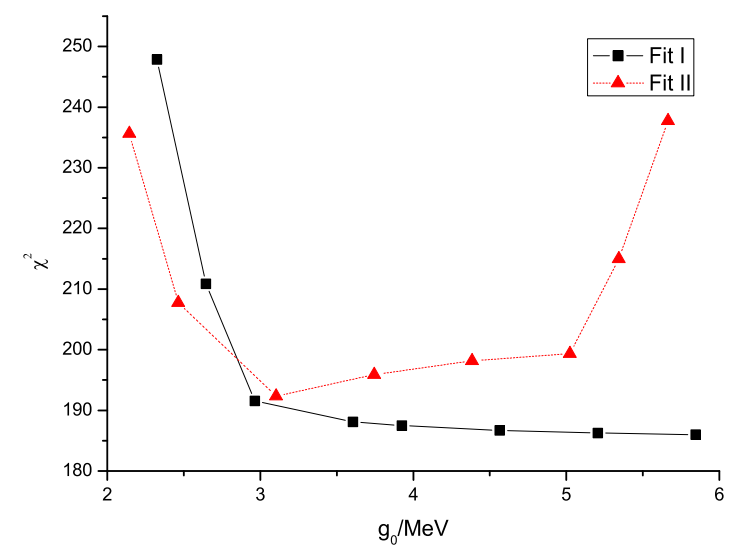

Figure 4: $\chi^{2}$ dependence on $g_{0}$. Fit I: without the $\omega \chi_{c 0}$ data, where we see the scaling behaviour; Fit II: with the $\omega \chi_{c 0}$ data where the scaling behaviour disappears.

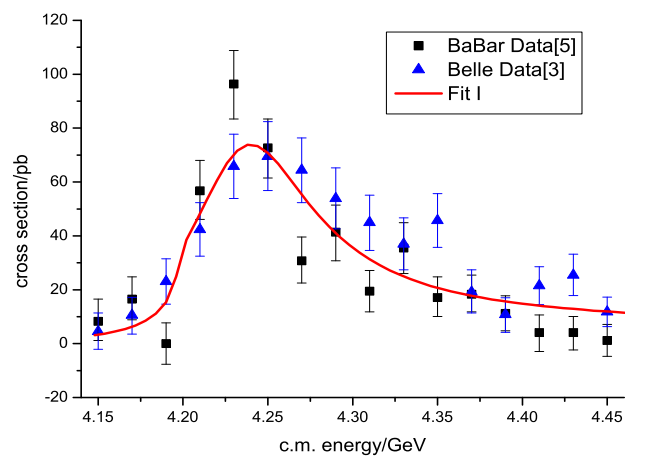

(a)

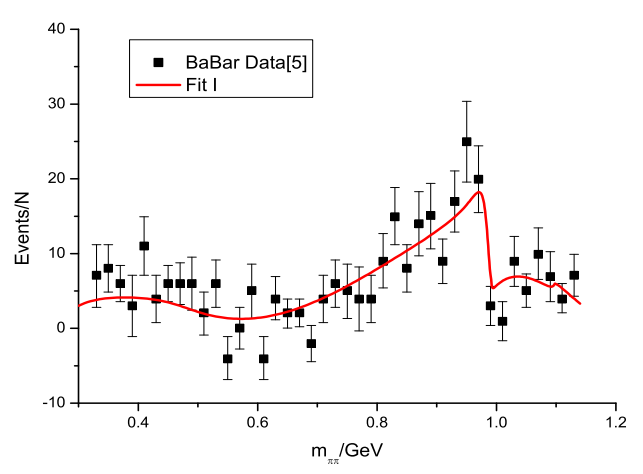

(c)

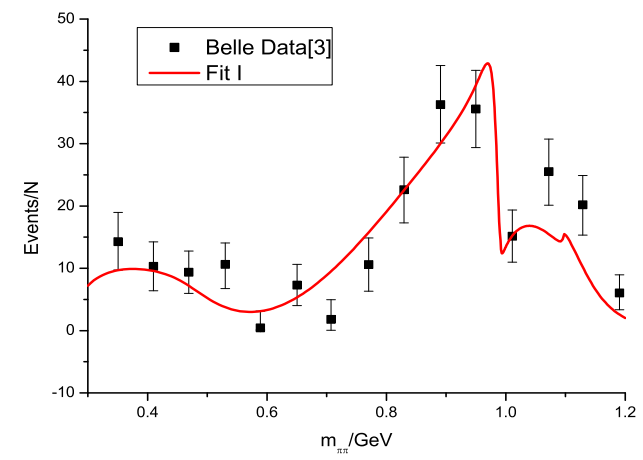

(b)

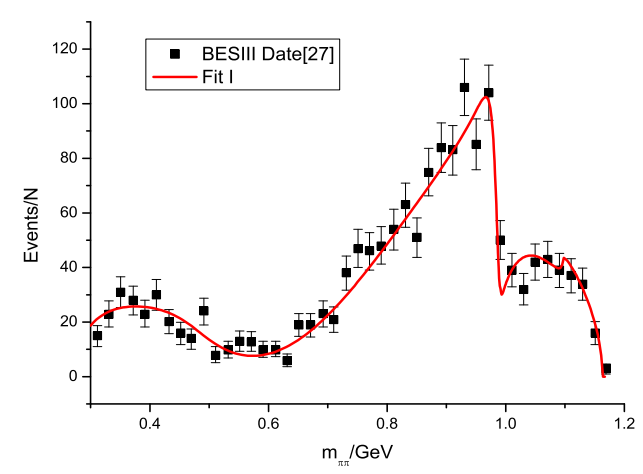

(d)

Figure 5: The fit to the cross section $e^{+} e^{-} \rightarrow J / \psi \pi \pi$ and $\pi \pi$ invariant mass spectrum without $\omega \chi_{c 0}$ data.

conservatively $<580 \mathrm{eV}$, at $90 \%$ confidence level. The dependence of $\chi^{2}$ on $g_{0}$ is clearly depicted in Fig. 4 (black square) which exhibits an approximate scaling law on parameter $g_{0}$. When $g_{0}$ increases, parameters $h_{i}$ have to become small to compensate for the experimental value of $\Gamma_{X \rightarrow J / \Psi \pi \pi}$ as given in Eq. (3). See Eqs. (11) and (11), it is easy to find out that $\Gamma_{X \rightarrow J / \Psi \pi \pi}$ is proportional to the product of $g_{0}$ and $h_{i}$. This mechanism keeps $\Gamma\left(q^{2}\right)$ of the denominator of $X(4260)$ propagator (see Eq. (17)) almost unchanged when $g_{0}$ varies. As a consequence, $\chi^{2}$ 


\begin{tabular}{c|c|c}
\hline & Fit I & Fit II \\
\hline$\chi^{2} /$ d.o.f. & $187.1 /(145-12)$ & $193.5 /(154-13)$ \\
\hline$g_{0}(\mathrm{MeV})$ & 4.24 (fixed) & $3.32 \pm 0.11$ \\
$g_{Y \omega \chi_{c 0}}$ & $0.17 \pm 0.01$ & $0.06 \pm 0.01$ \\
$M_{X}(\mathrm{GeV})$ & $4.2504 \pm 0.0034$ & $4.2432 \pm 0.0031$ \\
\hline
\end{tabular}

Table 2: Parameters from Fit I and Fit II. Ratio R is chosen at 0.66 for example. Since $g_{0}$ is fixed, the total parameters are 12 , and only important parameters are presented.

\begin{tabular}{c|cccc}
\hline \hline & sheet I & sheet II & sheet III & sheet IV \\
\hline$\Gamma_{J / \psi \pi \pi}+\Gamma_{h_{c} \pi \pi}$ & + & - & - & + \\
$\Gamma_{\omega \chi_{c 0}}$ & + & + & - & - \\
\hline \hline
\end{tabular}

Table 3: Definition of the four Riemann sheets with the $J / \psi \pi \pi\left(h_{c} \pi \pi\right)$ channel and $\omega \chi_{c 0}$ channel.

\begin{tabular}{c|clcl}
\hline \hline & sheet I & sheet II & sheet III & sheet IV \\
\hline Fit I & - & - & $4231.9-44.2 \mathrm{i}$ & $4233.2-42.5 \mathrm{i}$ \\
\hline Fit II & - & $4241.5-24.4 \mathrm{i}$ & $4232.8-36.3 \mathrm{i}$ & - \\
\hline
\end{tabular}

Table 4: Pole position of Fit I and II. The value of $\sqrt{s_{\text {pole }}}=M_{\text {pole }}-i \Gamma_{\text {pole }} / 2$ is given in MeV.

becomes almost inert with respect to the variation of $g_{0}$ when it is large enough since the effect can be counterbalanced by a variation of $h_{i}$. This observation is already made in V1, here we reconfirm the 'scaling law' even when $\Gamma_{h_{c} \pi \pi}$ is included.

The 'scaling law' means that we can not reliably determine parameter $g_{0}$ at all when $g_{0}$ is large enough. It is important to notice that in Fig. 4 there exists a large enough space for $g_{0}$ to maintain a (almost) minimal $\chi^{2}$, and at lower value it is below the BES $\Gamma_{X \rightarrow e^{+} e^{-}}$bound given in Ref. [43. As an example, we list the lowest value of $g_{0}$ at $4.24 \mathrm{MeV}$ which corresponds to $\Gamma_{X \rightarrow e^{+} e^{-}} \simeq 41.1 \mathrm{eV}$, and its fitting result is shown in Fig. [5 and Table 2 . For this chosen value, Table.2 indicates the widths of $J / \psi \pi \pi, h_{c} \pi \pi$ and $\omega \chi_{c 0}$ at $\sqrt{q^{2}}=4.26 \mathrm{GeV}$ are $32.4 \mathrm{MeV}$, 21.3 $\mathrm{MeV}$ and 49.8 $\mathrm{MeV}$, respectively.

We also search for poles of the $X(4260)$ propagator, and the complex plane is divided into four Riemann sheets by $\Gamma_{J / \psi \pi \pi}\left(\Gamma_{h_{c} \pi \pi}\right)$ and $\Gamma_{\omega \chi_{c 0}}$ defined in Table 3 , and the pole positions are presented in Table 4 It is noticeable that there are two pair of poles locating above the $\omega \chi_{c 0}$ threshold on the third and fourth sheet, and both of them show around $85 \mathrm{MeV}$ pole width for the $\mathrm{X}(4260)$ resonance. According to the pole counting rule in Ref. [44, 45], the two pair of near threshold poles indicate that $X(4260)$ is not like a molecule resonance but more like an 'elementary' particle or, in other words, a confining state.

For the purpose in extracting solid physical conclusions, we also varies the coefficient $\mathrm{R}$ as $0.56,0.46,0.36,0.26,0.16,0$ in the fit, and when $R=0$, the fit procedure is very similar with V1 [46], where we found a large coupling constant $g_{\omega \chi_{c 0}}$ and also the $\chi^{2}$ scaling law on $g_{0}$. It is found that qualitative physical results are not sensitive to the $R$ value, and all the fits with different $R$ maintain the same scaling law on the coupling constant $g_{0}$. In all cases the large coupling of the $\omega \chi_{c 0}$ to the $\mathrm{X}(4260)$ always exists which coincides with the prediction in V1.

Before going to the next section, a brief conclusion is in order: the fit result shows that the $\chi^{2}$ has an approximate scaling law on $g_{0}$, which could not be determined very well. Two nearby poles are found on the third and fourth Riemann sheet. The fit results are not sensitive to the $\mathrm{R}$ value, which indicates that $h_{c} \pi \pi$ only contributes to the $\mathrm{X}(4260)$ decay as a smooth background. We predict that the $\omega \chi_{c 0}$ channel has a large branching ratio in the $\mathrm{X}(4260)$ decay in all cases.

\subsection{Fit Including $\omega \chi_{c 0}$ Cross Section Data}

In the above subsection, we confirmed that there should be a sizable contribution from $\omega \chi_{c 0}$ channel even when the recent $\omega \chi_{c 0}$ cross section data from BESIII collaboration 33, 34, are not taken into consideration. In this subsection, we further include the $\omega \chi_{c 0}$ cross section data in 
our analysis to get a more precise result of $g_{\omega \chi_{c 0}}$. This will certainly benefit our understanding on $X(4260)$, provided that those $\omega \chi_{c 0}$ data indeed come from $X(4260)$ decay. The cross section of $e^{+} e^{-} \rightarrow X(4260) \rightarrow \omega \chi_{c 0}$ is parameterized as Ref. [4,

$$
\sigma_{e^{+} e^{-} \rightarrow X(4260) \rightarrow \omega \chi_{c 0}}\left(q^{2}\right)=\frac{3 \pi}{4 q^{2}} \frac{\Gamma_{e e} \Gamma_{\omega \chi_{c 0}}}{\left|D_{X}\left(q^{2}\right)\right|^{2}},
$$

where $\Gamma_{e e}$ is given in Eq. (2), $D_{X}\left(q^{2}\right)$ is the denominator of the $X(4260)$ propagator shown in Eq. (17) and (18) and $\Gamma_{\omega \chi_{c 0}}=g_{\omega \chi_{c 0}} k_{\omega \chi_{c 0}}$.

The major difference after taking the $\omega \chi_{c 0}$ data into Fit II, is that the $\chi^{2}$ scaling law on $g_{0}$ disappears. As shown in Fig团 (green triangle), the $\chi^{2}$ has the minimum at $g_{0} \simeq 3.32 \mathrm{MeV}$. It suggests that this fit is more stable than Fit I without the $\omega \chi_{c 0}$ cross section.

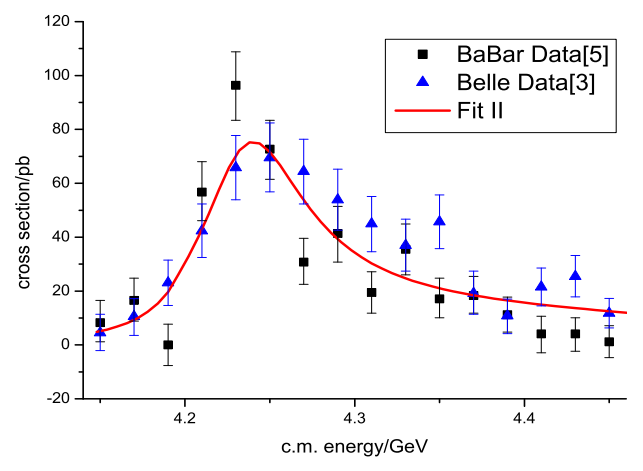

(a)

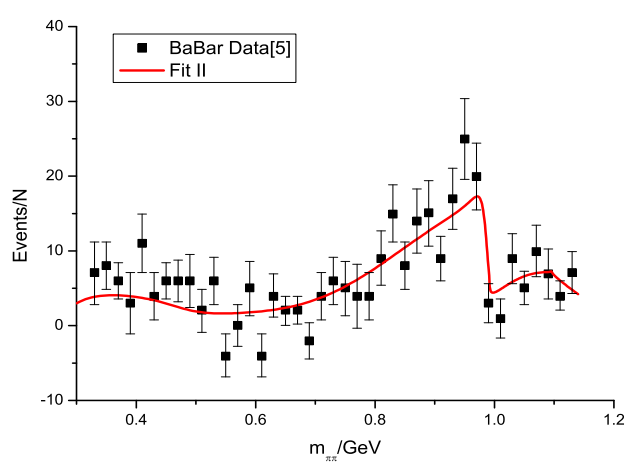

(c)

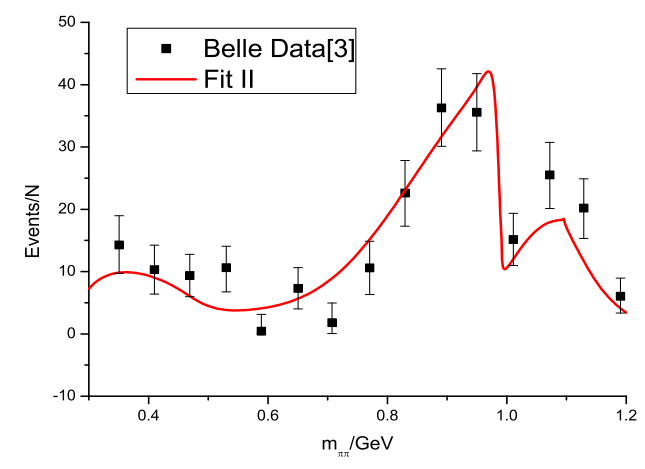

(b)

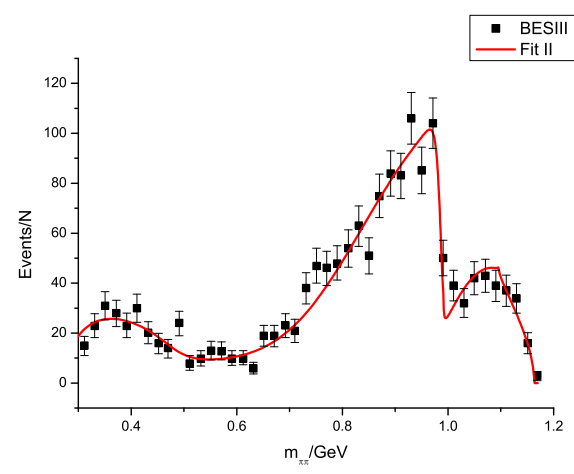

(d)

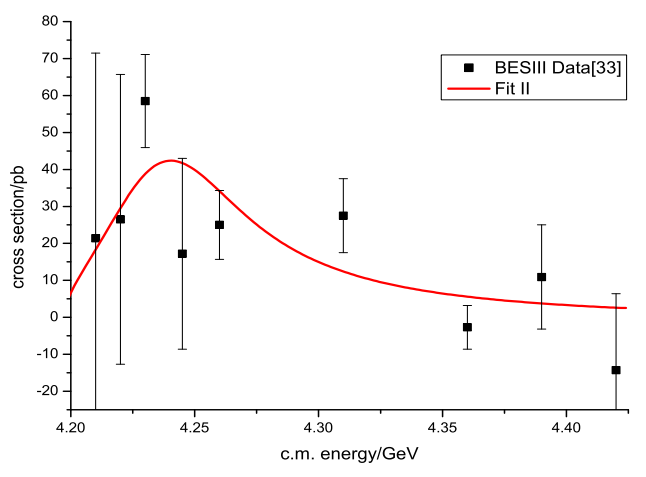

(e)

Figure 6: The cross section of $e^{+} e^{-} \rightarrow J / \psi \pi \pi$, invariant mass of $\pi \pi$, and the cross section of $e^{+} e^{-} \rightarrow \omega \chi_{c 0}$ 
We again take $R=0.66$ as the example, the fit result is shown in Fig.6] and Table[2] From Table. 2. one notices that the coupling of $\omega \chi_{c 0}$ approaches a smaller value (but still much larger than other open-charm channels), which is well constrained by the new data of $\mathrm{X}(4260)$ decaying into $\omega \chi_{c 0}$. The pole positions are also searched for and are shown in Table4. It should be noticed that there are two pairs of poles on sheet II and III, which has smaller pole width (around 60 $\mathrm{MeV}$ ) comparing with Fit I.

Comparing the $\chi^{2} / d o f$, Fit II (193.5/141) is similar in quality with that of Fit I $(187.1 / 133)$. The major different physical impact once the $\omega \chi_{c 0}$ data are included is that it roughly reduces $g_{\omega \chi_{c 0}}$ by a factor 3 . The three partial widths $\Gamma_{J / \psi \pi \pi}, \Gamma_{h_{c} \pi \pi}$ and $\Gamma_{\omega \chi_{c 0}}$ are now $45.1 \mathrm{MeV}$, $29.9 \mathrm{MeV}, 16.9 \mathrm{MeV}$ at $\sqrt{q^{2}}=4.26 \mathrm{GeV}$, respectively. The branching ratio of $\omega \chi_{c 0}$ in this fit is $18.5 \%$, which is still sizable, and is still much larger comparing with open-charm channels, such as $D \bar{D}, D^{*} \bar{D}^{*}$ and $D_{s}^{*} \bar{D}_{s}^{*}$. Furthermore, we verified that the qualitative result is not sensitive to the ratio $R$ ranging in $[0,0.66]$. See Table 3.3 for illustration.

\begin{tabular}{c|c|c|c|c|c|c|c}
\hline & \multicolumn{7}{|c}{ Fit II } \\
\hline \multicolumn{1}{c|}{$\mathrm{R}$} & 0.66 & 0.56 & 0.46 & 0.36 & 0.26 & 0.16 & 0.00 \\
\hline$\Gamma_{e e}$ & 25.23 & 24.36 & 23.55 & 22.82 & 22.19 & 22.03 & 22.02 \\
\hline$\Gamma_{\omega \chi_{c 0}}$ & 17.49 & 19.04 & 20.89 & 23.11 & 25.84 & 26.75 & 26.77 \\
\hline$\Gamma_{J / \psi \pi \pi}$ & 54.52 & 57.02 & 59.67 & 62.42 & 65.21 & 65.82 & 65.02 \\
\hline$\Gamma_{h_{c} \pi \pi}$ & 35.99 & 31.93 & 27.45 & 22.47 & 16.95 & 10.53 & 0 \\
\hline
\end{tabular}

Table 5: Partial widths obtained when $\mathrm{R}$ changes. The unit of $\Gamma_{e e}$ is in $\mathrm{eV}$ and the unit of the others is in $\mathrm{MeV}$.

For a summary, after taking the $\omega \chi_{c 0}$ cross section data into account, the coupling of the $\mathrm{X}(4260)$ decay into $\omega \chi_{c 0}$ becomes smaller but fixable, and even though it is no longer dominant but still plays an important role in the $X(4260)$ decay, which supports our conclusion before. Certainly, because of the large error bar near $4.26 \mathrm{GeV}$ from the $\omega \chi_{c 0}$ cross section data (in Fig. 6(e)) a more qualitative conclusion still needs more statistics from the experimental data.

\section{Conclusions and Discussions}

The property of $X(4260)$ remains mysterious after being discovered for almost ten years. The nature of this particle is still an controversial issue. In this work, we investigate this particle based on all experimental data available and very modest theoretical assumptions. Hence the conclusion we reached should be robust. Comparing with V1 [46], two more channels $h_{c} \pi \pi$ and $\omega \chi_{c 0}$ are now considered.

We have performed two fits: Fit I without and Fit II with the $\omega \chi_{c 0}$ cross section data. In the two scenarios, they have similar $\chi^{2} /$ dof but different behavior on the coupling constant $g_{0}$. In the former one a $\chi^{2}$ scaling law on $g_{0}$ is observed which could not be determined from the fit, while in the latter case the $\chi^{2}$ scaling law on $g_{0}$ disappears which has a minimum value when $g_{0}=3.32 \mathrm{MeV}$. The value of $g_{0}$ corresponds to $\Gamma_{e^{+} e^{-}} \simeq 25 \mathrm{eV}$, which is certainly below the $\Gamma_{e^{+} e^{-}}$bound in BES experiment [43]. Considering the variation of $\mathrm{R}$, we also give the following estimate on

$$
\Gamma_{e e}=23.30 \pm 3.55 \mathrm{eV}
$$

Compared with the experimental observation of $\Gamma_{e e} \times \operatorname{Br}(J / \psi \pi \pi)=9.7 \pm 1.1 \mathrm{eV}[3$ or $9.2 \pm 1.5 \mathrm{eV}$ [5], we conclude that the roughly half of $X(4260)$ decay into $J / \psi \pi \pi$ final state. Finally, our analysis points out a sizable coupling between $X(4260)$ and $\omega \chi_{c 0}$ which awaits a theoretical explanation.

In both fits there are two nearby poles found in $\mathrm{X}(4260)$ propagator indicating that the $X(4260)$ is most likely a confining state 44, 45. The small value of $\Gamma_{e^{+} e^{-}}$is consistent with the hybrid scenario which indicates $5.5 \pm 1.3 \mathrm{eV} \leq \Gamma_{e^{+} e^{-}} \leq 62 \pm 15 \mathrm{eV}$ [10] or $23 \pm 20 \mathrm{eV}$ [14. Also the hybrid state is suppressed to decay into $\bar{D} \bar{D}, D_{s} \bar{D}_{s}, D^{*} \bar{D}^{*}$ and $D_{s}^{*} \bar{D}_{s}^{*}$,47, which coincides with the experimental data for the $X(4260)$. Nevertheless, since $D_{1}$ is in $P$ wave, a hybrid state is likely to have a large coupling to $D D_{1}$ channel, this is not supported by experiment 48, and 
our analysis. It should be pointed out that a small value of $\Gamma_{e^{+} e^{-}}$is also consistent with the explanation that $X(4260)$ is the $3 \mathrm{D}$ charmonium state. The difficulty of this possible explanation comes from the role of $X(4160)$, which is considered as candidate of the 3D charmonium state in the literature, though it has a rather large $\Gamma_{e^{+} e^{-}}$width. We hope our effort made in this paper will be helpful for future investigations in clarifying the issue of $X(4260)$.

\section{Acknowledgement}

We are grateful to illuminating discussions with Chang-Zheng Yuan and Kuang-Ta Chao, and would also like to thank Gui-Jun Ding, Ce Meng, Qiang Zhao and Bing-Song Zou for helpful discussions. This work is supported in part by National Nature Science Foundations of China under contract number 10925522 and 11021092.

\section{Reference}

[1] BABAR Collaboration, B. Aubert et al., Phys. Rev. Lett. 95, 142001 (2005).

[2] CLEO Collaboration, T. E. Coan et al., Phys. Rev. Lett. 96, 162003 (2006).

[3] BELle Collaboration, C. Z. Yuan et al., Phys. Rev. Lett. 99, 182004 (2007).

[4] Particle Data Group, K. A. Oilve et al., Chin. Phys. C 38, 090001 (2014).

[5] BaBar Collaboration, J. Lees et al., Phys. Rev. D 86, 051102 (2012).

[6] S. Godfrey and N. Isgur, Phys. Rev. D 32, 189 (1985).

[7] E. S. Swanson, Phys. Rept. 429, 243 (2006).

[8] X. Liu, X. Q. Zeng, and X. Q. Li, Phys. Rev. D 72, 054023 (2005).

[9] C. Z. Yuan, P. Wang, and X. H. Mo, Phys. Lett. B 634, 399 (2006).

[10] F. E. Close and N. Page, Phys. Lett. B 628, 215 (2005).

[11] S. L. Zhu, Phys. Lett. B 625, 212 (2005).

[12] F. Buisseret and V. Mathieu, Eur. Phys. J. A 29, 343 (2006).

[13] E. Kou and O. Pene, Phys. Lett. B 631, 164 (2005).

[14] Y. Chen et al., PoS LATTICE2013, 251 (2014).

[15] C. F. Qiao, Phys. Lett. B 639, 263 (2006).

[16] J. L. Rosner, Phys. Rev. D 74, 076006 (2006).

[17] G. J. Ding, Phys. Rev. D 79, 014001 (2009).

[18] Q. Wang et al., Phys. Rev. D 89, 034001 (2014).

[19] E. van Beveren, G. Rupp, and J. Segovia, Phys.Rev.Lett. 105, 102001 (2010).

[20] D.-Y. Chen, J. He, and X. Liu, Phys. Rev. D 83, 054021 (2011).

[21] R. Albuquerque, F. Fanomezana, S. Narison, and A. Rabemananjara, Nucl. Phys. Proc. Suppl. 234, 158 (2013).

[22] L. Maiani et al., Phys. Rev. D 87, 111102 (2013).

[23] J.-R. Zhang and M.-Q. Huang, Phys. Rev. D 83, 036005 (2011).

[24] D. Ebert, R. Faustov, and V. Galkin, Eur. Phys. J. C 58, 399 (2008).

[25] D. Ebert, R. Faustov, and V. Galkin, Physics of Atomic Nuclei 72, 184 (2009). 
[26] L. Maiani, F. Piccinini, A. Polosa, and V. Riquer, Phys. Rev. D 72, 031502 (2005).

[27] BESIII Collaboration, M. Ablikim et al., Phys. Rev. Lett. 110, 252001 (2013).

[28] Belle Collaboration, Z. Liu et al., Phys. Rev. Lett. 110, 252002 (2013).

[29] T. Xiao, S. Dobbs, A. Tomaradze, and K. K. Seth, Phys. Lett. B 727, 366 (2013).

[30] BABAR Collaboration, B. Aubert et al., Phys. Rev. D 79, 092001 (2009).

[31] Belle Collaboration, G. Pakhlova et al., Phys. Rev. D 77, 011103 (2008).

[32] Belle Collaboration, G. Pakhlova et al., Phys. Rev. Lett. 98, 092001 (2007).

[33] C.-P. Shen, Talk given at Workshop on Exotic States, July 12-16, 2014, Rizhao, China .

[34] BESIII Collaboration, M. Ablikim et al., (2014), 1410.6538.

[35] BESIII Collaboration, M. Ablikim et al., Phys. Rev. Lett. 111, 242001 (2013).

[36] S. U. Chung, Phys. Rev. D 48, 1225 (1993).

[37] K. L. Au, D. Morgan, and M. R. Pennington, Phys. Rev. D 35, 1633 (1987).

[38] L. Y. Dai, X. G. Wang, and H. Q. Zheng, Commun. Theor. Phys 57, 841 (2012).

[39] Y. Mao et al., Phys. Rev. D 79, 116008 (2009).

[40] Z. Y. Zhou et al., JHEP 0502, 043 (2005), hep-ph/0406271.

[41] A. G. Nicola and J. R. Pelaez, Phys. Rev. D 65, 054009 (2002).

[42] C.-Z. Yuan, Chin. Phys. C 38, 043001 (2014).

[43] X. H. Mo et al., Phys. Lett. B 640, 182 (2006).

[44] D. Morgan, Nucl. Phys. A 543, 632 (1992).

[45] O. Zhang, C. Meng, and H. Q. Zheng, Phys. Lett. B 680, 453 (2009).

[46] L. Dai, M. Shi, G.-Y. Tang, and H. Zheng, (2012), arXiv:1206.6911[hep-ph].

[47] P. R. Page, E. S. Swanson, and A. P. Szczepaniak, Phys. Rev. D 59, 034016 (1999).

[48] BESIII Collaboration, M. Ablikim et al., Phys.Rev.Lett. 112, 022001 (2014). 